\section{Role of Structural Homo- and Hetero- zygosity in Mosaic Formation}

AN investigation into the nature of mosaicism and the regularities of inheritance of mosaic characters in Drosophila melanogaster has revealed two essentials. Mosaicism of the ever-sporting displacement type is always connected with a chromosome re-arrangement involving heterochromatin regions of chromosomes. Insertion of extra heterochromatin of $X$ - or $Y$-chromosome or arms of the latter causes strong suppression of mosaicism.

As my investigations have shown, one of the peculiarities of heterochromatin action in mosaic formation is the maternal effect, that is, extra heterochromatin of the mother greatly decreases the development of mosaic individuals among those of her offspring that have no extra heterochromatin. Further investigations have now shown that the influence of heterochromatin in mosaic formation also exists in the form of a paternal effect. The two types of influence are not specific for any one mosaic strain but are inherent in all mosaic forms of the type of ever-sporting displacements.

The discovery of the paternal and maternal effect of heterochromatin in mosaic formation as well as the uniformity of its action was sufficient to suggest some general mechanism for these phenomena. The existing point of view according to which the action of heterochromatin is to be considered as the result of the breaking down of the quantitative balance between the hetero- and eu-chromatin of the chromosomes of the nucleus is not in agreement with these results.

By studying the influence of different heterochromatin components of chromosomes in a number of mosaic strains, I have collected a large body of data which has made it possible to analyse the regularities of the influence of heterochromatin in mosaic formation. The regularities found are as follows: (1) All heterochromatic components of chromosomes are able to suppress the development of mosaic characters. (2) The influence of extra heterochromatic components in mosaic development is found to exist in the form of a maternal, paternal or immediate (direct) effect: The results of the last-mentioned may be complicated by the additive influence of maternal or paternal effects. (3) Simple quantitative relations of heterochromatin action in mosaicism take place only in the case of the immediate effect when maternal or paternal effects are absent. When these latter are involved, simple quantitative regularity of heterochromatin influence in mosaicism breaks down. (4) Maternal and paternal effects do not follow from simple quantitative relations of hetero- and euchromatin, their effect being absent or present even if the quantitative relations of hetero- and euchromatin are the same. Only in the case of structural homozygosity of heterochromatin components of the chromosome does the maternal or paternal effect of the influence of heterochromatin in mosaicism take place. In the case of structural heterozygosity both are absent.

Structural homozygosity, in my opinion, means a structural identity in relation to heterochromatin components of chromosomes that secures the complete conjugation of the chromosome during meiosis and possibly during mitosis. It is necessary to emphasize that the term 'structural homozygosity' does not necessarily imply recognition of the pairing of heterochromatin regions, but only recognition of the possibility of their conjugation.
The influence of heterochromatin components of chromosomes in mosaicism in the form of maternal or paternal effects is transmitted from the parents to their offspring by the heterochromatin components themselves, no matter whether heterochromatin is present from a $Y$-chromosome or from one of its arms, from an $X$-chromosome or any autosome or from the heterochromatin of a re-arranged chromosome or scute-eight type.

Under the influence of structural homo- and heterozygosity there take place qualitative changes of chromosomes depending upon intracellular processes. I have shown that in homozygous scute-eight females, genetic differences between $X$-chromosomes can be observed. A chromosome transmitted by the father, owing to its structural heterozygosity, is qualitatively different from one transmitted by the mother.

My genetic studies were verified by cytological investigations (by Prokofieva-Belgovskaya). According to these, one of the $X$-chromosomes in homozygous scute-eight females has a heterochromatized yellowachæte region, while in the opposite $X$-chromosome this region is euchromatized. These differences may go so far as to make the distal ends of the $X$-chromosome unable to conjugate at all.

Quantitative changes of chromosomes resulting from the influence of structural homo- and heterozygosity are not connected only with mosaic strains. They arise in every stock where the appropriate conditions above mentioned are present. Owing to the instability of its features, mosaicism is only a sensitive indicator, allowing us to detect these qualitative changes of chromosomes.

Since extra heterochromatin leads to euchromatization, it is possible to say that structural homozygosity leads also to euchromatization of chromosomes, while structural heterozygosity leads to heterochromatization of their parts. It is the cause of increased fre. quency of mosaic individuals under conditions of structural heterozygosity and decreased frequency under conditions of structural homozygosity in genetic structures of type scute-eight. I believe that the phenomenon of heteropycnosis which heterochromosomes undergo in spermatogenesis is the result of structural heterozygosity of $X-Y$ composition. $X X$ composition, being of homozygous structure, does not show heteropycnosis during oogenesis.

Using the phenomenon of structural homo- and heterozygosity in chromosome changes, I have obtained stable changes that have been transmitted during many generations without any decreasing effect.

The data described above enable us not only to understand the nature of mosaicism and the nature of the influence of heterochromatin in mosaicism but also to regard structural homo- and heterozygosity as one of the essential intracellular factors responsible for causing stable changes in chromosomes and their genetic peculiarities. This throws new light on the problem of hybridization, as a factor of variability responsible not only for recombinations of parental, characters, but also for new qualitative changes. The importance of the latter is enhanced in the light of recent data on the presence of intrachromosome inert regions, and on the role of chromosome rearrangements among natural populations.

Academy of Sciences, N. J. NoUJnIN. Moseow. 